\title{
The Convex Polytopes and Homogeneous Coordinate Rings of Bivariate Polynomials
}

\author{
Shamsatun Nahar Ahmad ${ }^{1 *}$, Nor'Aini Aris ${ }^{2}$, Azlina Jumadi ${ }^{1}$ \\ ${ }^{1,3}$ Faculty of Computer and Mathematical Sciences, Universiti Teknologi MARA \\ 85000 Segamat, Johor, Malaysia \\ ${ }^{2}$ Mathematical Sciences Department, Faculty of Sciences, Universiti Teknologi Malaysia \\ 81310 Skudai, Johor, Malaysia \\ *Corresponding author e-mail: shams551@uitm.edu.my
}

Received: 29 July 2019

Accepted: 25 September 2019

First Online: 30 November 2019

\section{ABSTRACT}

Concepts from algebraic geometry such as cones and fans are related to toric varieties and can be applied to determine the convex polytopes and homogeneous coordinate rings of multivariate polynomial systems. The homogeneous coordinates of a system in its projective vector space can be associated with the entries of the resultant matrix of the system under consideration. This paper presents some conditions for the homogeneous coordinates of a certain system of bivariate polynomials through the construction and implementation of the Sylvester-Bèzout hybrid resultant matrix formulation. This basis of the implementation of the Bèzout block applies a combinatorial approach on a set of linear inequalities, named 5-rule. The inequalities involved the set of exponent vectors of the monomials of the system and the entries of the matrix are determined from the coefficients of facets variable known as brackets. The approach can determine the homogeneous coordinates of the given system and the entries of the Bèzout block. Conditions for determining the homogeneous coordinates are also given and proven.

Keywords: algebraic geometry, Bèzout resultant matrix, combinatorial, facet variable, homogeneous coordinates 


\section{INTRODUCTION}

The fundamental objects of study in algebraic geometry are algebraic varieties, which are geometric manifestations of solutions of systems of polynomial equations. The studied classes of algebraic varieties were in plane algebraic curves, which include lines and circles, and toric varieties such as divisors and fans (collection of cones) as in Puenta [1] and Karzhemanov [2,3]. A good reference in the field of algebraic geometry can be referred in Cox et. al $[4,5]$. Basically, the subject of algebraic geometry is the study of systems of polynomial equations in several variables, that has a wide range of applications in science and engineering, for instance in robotic, camera motion, computer aided design (CAD) and computer aided manufacturing (CAM) systems [6]. Those systems were modeled as sparse polynomials which can be utilised by considering the combinatorial structure of the polynomials represented as the Newton polytopes, refer Cox et. al [7], which is the convex set of the exponent vectors of the polynomials' variables. Recently, Dickenstein et. al [8] also worked on the combinatorics of polytopes but in 4-dimensional.

Ahmad and Aris [9] presented an algorithm that has applied the concepts from algebraic geometry to determine the homogeneous coordinate ring of bivariate polynomials and to compute the entries of the Bèzout matrix of the system. The algorithm terminates successfully with the correct matrix dimension and entries in comparison with the work given in Khetan [10]. In this paper, we study the effects of translating the vertices of the polytopes of the polynomial system studied in Khetan. The effects of scaling the dimension of the Newton polytope are also investigated. Thus, sufficient conditions in deriving the homogeneous coordinates of such systems will be established.

\section{PRELIMINARIES ON ALGEBRAIC GEOMETRY}

Considering an unmixed polynomial system whose supports are identical in the following form:

$$
f_{i}:=\sum_{\alpha \in A} C_{i \alpha} x^{\alpha}
$$


With $i=1, \ldots, n+1$. Let $f_{1}, \ldots, f_{n+1} \in \mathbf{C}\left[x_{1}, \ldots x_{n}\right]$ be $n+1$ polynomials in $n$ variables with the same Newton polytope $Q \subset \mathbf{R}^{n}$ which is known as a convex hull of the supports, $Q=\operatorname{conv}(\mathrm{A})$ and $A=Q \cap \mathbf{Z}^{n}=\left\{\alpha_{1}, \ldots, \alpha_{N}\right\}$ is a set of the supports of the polynomials affinely span $Z^{\mathrm{n}}$. Note that $\mathrm{R}$ is the set of real numbers, $\mathrm{Z}$ is the set of integer numbers and both sets are in the affine space $\mathbf{C}^{\mathrm{n}}$.

The interesting type of hybrid matrix for this research is as the following:

$$
\left(\begin{array}{cc}
B e z & S y l^{*} \\
S y l & 0
\end{array}\right)
$$

Where the entries of $S y l$ and $S y l^{*}$ are of Sylvester type, the entries of $B e z$ are of Bèzout type and 0 is a square matrix of zero entries. In this paper, the application of algebraic geometry is focused only on the Bèzout block. The entries of the Bèzout block are linear forms in the bracket variables [uvw] defined as follows:

$$
[\mu v \gamma]=\left|\begin{array}{lll}
C_{1 \mu} & C_{1 v} & C_{1 \gamma} \\
C_{2 \mu} & C_{2 v} & C_{2 \gamma} \\
C_{3 \mu} & C_{3 v} & C_{3 \gamma}
\end{array}\right|
$$

Where $C_{i \mu}, C_{j v}, C_{k \gamma}$ are the coefficients $x^{\alpha} \in f_{i}$ of. The bracket variables satisfy a certain set of inequalities formulated by Khetan [10] and were implemented to five rules by Ahmad and Aris [9], named 5-Rule and was computed with complexity $\mathcal{O}\left(n^{4}\right)$.

$$
\begin{aligned}
& \text { Rule1: } \forall k \in R_{3}, \gamma_{k}>a_{k} \\
& \text { Rule2: } \exists j \in R_{2}, \gamma_{j} \leq a_{j} \\
& \text { Rule3: } \forall j \in R_{2}, v_{j}+\gamma_{j}>a_{j} \\
& \text { Rule4: } \exists_{i} \in R_{1}, v_{i}+\gamma_{i} \leq a_{i} \\
& \text { Rule5: } \forall i \in R_{1}, \mu_{i}+v_{i}+\gamma_{i}>a_{i}
\end{aligned}
$$


Where $\mathrm{R}_{1}, \mathrm{R}_{2}$ and $\mathrm{R}_{3}$ are the similar partitions of the fan constructed in Khetan [10]. In addition, Khetan's formula of the Bèzout matrix is also considered and restated in the following theorem.

Theorem 1. The Bèzout matrix is the matrix of the linear map $\mathrm{T}_{Q}:\left(S_{Q}\right)^{*} \rightarrow S_{\mathrm{int}(2 Q)}$ defined by

$$
\mathrm{T}_{Q}\left[\left(y^{\alpha}\right)^{*}\right]=\sum_{(\mu, \nu, \lambda) \in F_{\alpha} \subset A^{3}}[\mu v \gamma] y^{\mu+v+\gamma-\alpha-\omega_{0}}
$$

$\omega_{0}=(1,1, \ldots, 1)$, and $F_{\alpha}$ is the set of all triples $(\mu, v, \gamma) \in \phi_{Q}(A)^{3}$ satisfying (4).

The bracket variables $[\mu v \gamma]$ in Theorem 1 , are also known as the coefficients of the facet variables for the polynomial ring and the exponent vectors of the facet variables as follows,

$$
\phi_{Q}\left(x^{\alpha}\right)=y^{\alpha}=\prod_{i=1}^{s} y_{i}^{\phi_{Q}(\alpha)} .
$$

From the formulation in Theorem 1, it will produce a homogeneous polynomial, and the bracket $[\mu v \gamma]$ is denoted as coefficients of facet variables $y_{1}, y_{2}, \ldots, y_{s}$ while $\mu+v+\gamma-\alpha-\omega_{0}$ is the formula to enumerate the exponents for each facet variable. Sum of the exponents must equal for each monomial of the homogeneous polynomials with bracket coefficients. Therefore, one can be written as follows,

$$
\phi_{Q}(\alpha)_{i}=\left\langle\alpha, v_{i}\right\rangle+a_{i} \cong \mu_{i}+v_{i}+\gamma_{i}-\alpha_{i}-\omega_{0} \text { for } i=1, \ldots, s .
$$

In the next definition is to define projective space. This space is very important for homogeneous polynomial system.

$$
P^{n}(K)=\left(K^{n+1}-\{0\}\right) / \sim
$$

Definition 1. [7] Given the set of n-dimensional projective space where each nonzero $(n+1)$ - tuple, $\left(x_{0}, \ldots, x_{n}\right) \in K^{n+1}$ defines a point $p \in \mathbf{P}^{n}(K)$ an $\left(x_{0}, \ldots, x_{n}\right)$ is called the homogeneous coordinate of $p$. 
Geometrically the polynomial (1) can be viewed as the Newton polytope $Q \subset \mathbf{R}^{n}$ and support $A=Q \cap \mathbf{Z}^{n}=\left\{\alpha_{1}, \ldots, \alpha_{N}\right\}$. The support can be plot in a Cartesian coordinate, $(x, y)$ if the dimension of affine space is 2 and it is called polytope. Thus, in this implementation, the polynomial equations with two variables and three equations are used. Polytopes have faces, edges and vertices. The faces of polytope are when polygons lying in plane, while edges are line segments that connect certain pairs of vertices (faces of dimension 1) and vertices are points (faces of dimension 0 ). Hence, each facet $F=\left\{\tau_{1}, \ldots, \tau_{s}\right\}$ of $Q$ is a polytope of dimension less than dimension of $Q$. Therefore, if $Q$ has dimension $n$, then facets are faces of dimension $n-1$.

To define a face of an arbitrary Newton polytope, an affine hyperplane is needed. Let $v$ be a nonzero vector in $\mathbf{R}^{n}$, an affine hyperplane is defined by an equation of the form $m . v=-\mathrm{a}$. Therefore, for every unmixed system of polynomial equations, there exists an associated Newton polytope $Q$ defined by

$$
a_{i}=-\min _{m \in Q}\langle m \cdot v\rangle
$$

Thus, any Newton polytope $Q$ in $\mathbf{R}^{n}$ with s number of edges can be defined by its facet inequalities given by

$$
Q=\left\{m \in R^{n} \mid\left\langle m, v_{i}\right\rangle \geq-a_{i}, i=1, \ldots, s\right\},
$$

for some integers $a_{1}, \ldots, a_{\mathrm{s}}$ which are referred to as the data for $Q$. The inner normal $v_{1}, \ldots, v_{\mathrm{s}}$ are called rays. The set of data and inner normal are unique for the facets. Hence the following definition are needed for homogenisation coordinate $x^{\mathrm{a} 1}, \ldots,{ }^{\text {as }}$.

Definition 2. [10] The Q-homogenisation map $\phi_{Q}: \mathbf{Z}^{n} \rightarrow \mathbf{Z}^{s}$ is defined by for $i=1, \ldots, s$.

Definition 3. [10] The homogeneous coordinate ring for $X=X_{A}$ is the polynomial ring $S_{X}=K\left[y_{1}, \ldots, y_{s}\right]$ such that the monomials are graded.

Let $\mathrm{S}$ be the polynomial ring, $\mathbf{S}=\mathbf{C}\left[\mathrm{y}_{1}, \ldots, \mathrm{y}_{\mathrm{s}}\right]$ with one variable for each ray in the fan $\sum Q$ determining the toric variety $X_{\mathrm{A}}$. 
Let $Q \subset \mathbf{R}^{n}$ be the Newton polytope of dimension $n$, and let the support $A=\left\{\alpha_{1}, \ldots, \alpha_{N}\right\}$ such that convex $A$ equals $Q$. The toric variety $X_{A}=\overline{\phi_{A}\left(\left(\mathbf{C}^{*}\right)^{n}\right)} \subset \mathbf{P}^{N-1}$ is the dimension $\mathrm{n}$ variety defined as the Zariski closure of the set $\left\{x^{\alpha_{1}}, \ldots, x^{\alpha N}\right\}$ that is the image of $\phi_{A}$ where $\alpha_{i} \in \mathbf{Z}^{n}$; and $x=\left(x_{1}, \ldots, x_{\mathrm{n}}\right) \in\left(\mathbf{C}^{*}\right)^{n}$. Precisely the elements of $\phi_{A}$ are described in the following,

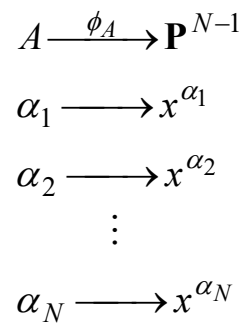

For all facets, the set of cones $\sigma_{\tau}$ are generated by inner normals. Then $\sum_{Q}=\left\{\sigma_{\tau} \mid \tau\right.$ is a face of $\left.Q\right\}$ is the normal fan of $Q$. This gives a toric variety denoted $X_{A}$. Each vertex of the cones is spanned by the inner normal $v_{i}$ corresponding to facets (edges for two dimension) which are incident to the vertex. The characterisation of the normal fan is stated in the following theorem.

Theorem 2. [4] The normal toric variety of a fan $\sum_{O} \in \mathbf{R}^{n}$ is projective if and only if $\sum Q$ is the normal fan of an n-dimensional lattice polytope in $\mathbf{R}^{n}$.

Besides a complete characterisation of polytope $Q$ in terms of the rays in its normal fan, Weil divisors are describing in the following proposition.

Proposition 3. [10] The $v_{i}$ are in one to one correspondence with the T-invariant prime Weil divisors on $X_{A}$. Di denotes the divisor corresponding to $v_{i}$.

A divisor $\mathrm{D}=\sum a_{i} D_{i}$ determines a convex polytope of (6). The divisor is best described as a line bundle and a global section of that line bundle. 
The following example is to show an edge and its inward normal of a Newton polytope in $\mathbf{R}^{2}$ that defines a hyperplane $\langle m, v\rangle=-a$.

Example 1. Consider a face of Newton polytope in $\mathbf{R}^{2}$ given in Figure 1. The edge $\mathrm{AB}$ joins the vertex $A(0,1)$ and $B(1,2)$, defines a hyperplane $\langle m, v\rangle=-a$ where $m$ is any point on $A B$ and $v=(1,-1)$ is an inward normal to $A B$.

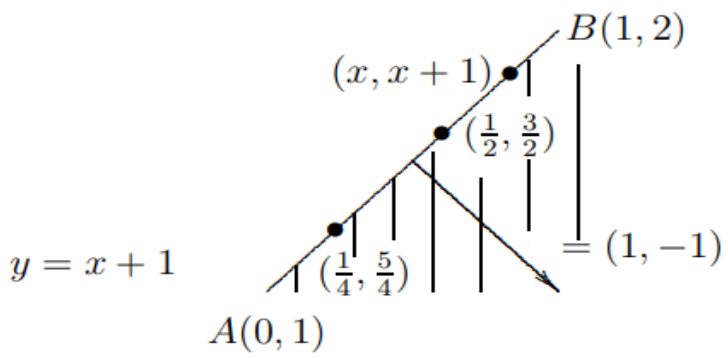

Figure 1: An Edge an Inward Normal

Since $m$ lies on $A B, m(x, x+1)$. Therefore $\langle m, v\rangle=\langle(x, x+1),(1,-1)\rangle=$ $x-x-1=-1$. Here $a=1$. For instance, $\left(\frac{1}{2}, \frac{3}{2}\right)$ is on $A B$ giving $\left\langle\left(\frac{1}{2}, \frac{3}{2}\right),(1,-1)\right\rangle=-1$. In addition, any point any point $m$ ' in the shaded region satisfies the equation $\left.\left\langle m^{\prime}, v\right\rangle\right\rangle-1$.

\section{IMPLEMENTATION OF THE BÈZOUT BLOCK}

Considering $(0,0)$ as an exponent vector (the constant term being non zero), a generalisation for the unmixed system worked by Khetan [10], we preserved the geometric structure, gives the system:

$$
f_{i}=C_{i 1} x^{0} y^{0}+C_{i 2} x^{h} y^{0}+C_{i 3} x^{0} y^{k}+C_{i 4} x^{h} y^{k}+C_{i 5} x^{2 h} y^{k}+C_{i 6} x^{h} y^{2 k}
$$

with $i=1,2,3$, and $A .\{(0,0),(h, 0),(0, k),(h, k),(2 h, k),(h, 2 k)\}$ Geometrically, by Example 1, the Newton polytope for the unmixed polynomial system (7) can be illustrated as in Figure 2. 


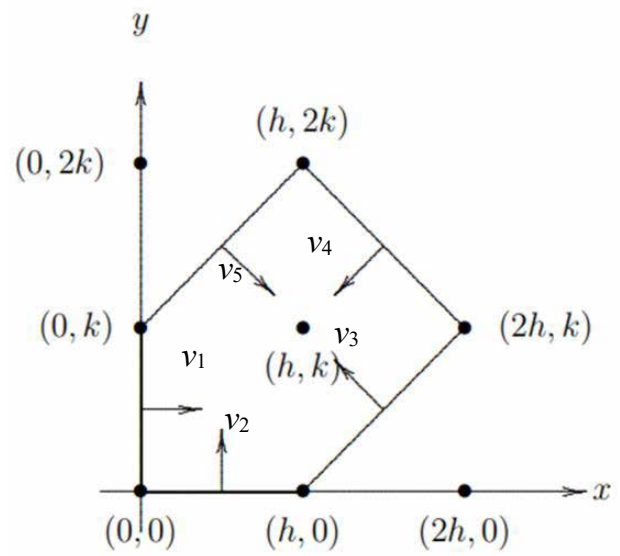

Figure 2: Translated Newton Polytope of System (7)

In this paper, the application of algebraic geometry is discussed with respect to the computation of all valid combinations of $(\mu, v, \lambda)$ that satisfy the inequalities in 5-Rule (4) and to compute the respective degree of the facet variables defined by the polynomial equations (7).

The vertices of the Newton polytope $Q$ shown in Figure 2 are sorted in counter clockwise, starting at the origin $(0,0)$ as a distinguish of point. The direction of sorting the vertices is fixed to obtain an appropriate pair of a vertex and its ray which gives the hyperplane data computation of the Newton polytope. The hyperplane data is written as $a_{1}, \ldots, a_{\mathrm{s}}$ and is defined by the equation of hyperplane (5), so that $D_{\mathrm{Q}}=\sum a_{i} D_{i}$ is the corresponding divisor. These divisors are the elements of the vector space $\mathrm{S}_{Q}$.

Suppose $h=k=1$, the computation of the partition of the fan, $R_{1}, R_{2}$ and $R_{3}$ are derived as in Table 1. Thus, the sets of partition of the fans are $R_{1}=\{1,5\}, R_{2}=\{2,3\}$ and $R_{3}=\{4\}$. Each vertex of the Newton polytope gives a two-dimensional cone in the normal fan where the rays, $v$ is the generator of the cones. By gluing of cones, the resulting normal fan of the translated Newton polytope (Figure 2) is shown in Figure 3. This fan gives $\mathbf{P}^{4}$, such that all two-dimensional cones $\sigma_{1}, \sigma_{2}, \sigma_{3}, \sigma_{4}, \sigma_{5}$ are generated by the bases of $\mathbf{Z}^{2}$. This implies that the affine toric varieties $X_{\sigma_{i}}$ are copies of $\mathbf{C}^{2}$. By gluing together five copies of $\mathbf{C}^{2}, \mathbf{P}^{4}$ is constructed. For simplicity, each ray is represented by numbers $1,2,3,4,5$. Hence, the partitions of the 
fan for the case $h=k=1$ is viewed in Figure 3 by applying the concept of cones and fan.

Table 1: Partition of the Fan $\boldsymbol{R}_{\boldsymbol{i}}$

\begin{tabular}{|c|c|c|}
\hline Partition $R_{i}$ & $i$ & Inward normal $v_{i}$ \\
\hline $\begin{array}{l}R_{1}=\left\{i: v_{i}=c_{1} v_{1}+c_{2} v_{2}\right\} \\
\text { with } c_{1} \geq 0 \text { and } c_{2} \leq 0\end{array}$ & 1 & $\begin{array}{l}v_{1}=c_{1}(1,0)+c_{2}(0,1)=(1,0) \\
c_{1}=1, c_{2}=0 \\
v_{5}=c_{1}(1,0)+c_{2}(0,1)=(1,-1) \\
c_{1}=1, \quad c_{2}=-1\end{array}$ \\
\hline $\begin{array}{l}R_{2}=\left\{i: v_{i}=c_{1} v_{1}+c_{2} v_{2}\right\} \\
\text { with } c_{1} \leq 0 \text { and } c_{2} \geq 0\end{array}$ & 2 & $\begin{array}{l}v_{2}=c_{1}(1,0)+c_{2}(0,1)=(0,1) \\
c_{1}=0, c_{2}=1 \\
v_{3}=c_{1}(1,0)+c_{2}(0,1)=(-1,1) \\
c_{1}=-1, \quad c_{2}=1\end{array}$ \\
\hline $\begin{array}{l}R_{3}=\left\{i: v_{i}=c_{1} v_{1}+c_{2} v_{2}\right\} \\
\text { with } c_{1}<0 \text { and } c_{2}<0\end{array}$ & 4 & $\begin{array}{l}v_{4}=c_{1}(1,0)+c_{2}(0,1)=(-1,-1) \\
c_{1}=-1, c_{2}=-1\end{array}$ \\
\hline
\end{tabular}

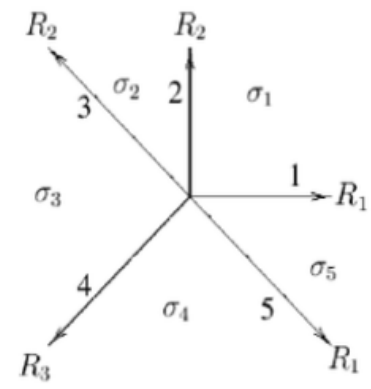

Figure 3: The Fan of Figure 2 When $h=k=I$

The above fan is determined by the divisor $D=\sum_{i=1}^{5} a_{i} D_{i}=D_{3}+$ $3 D_{4}+D_{5}$ such that $a_{1}=0, a_{2}=0, a_{3}=1, a_{4}=3, a_{5}=1$ and generates by $v_{1}, v_{2}, v_{3}, v_{4}, v_{5}$.

The formulation of the 5-Rule involves combinatorial approach where all the nonzero coefficients in the polynomials are considered and it applied to the structure of the Newton polytope of the associated polynomial system (7). In order to obtain the efficiency and effectiveness in generating the 
bracket variables $[\mu v \gamma](3)$ for the entries of the Bèzout block, we formulated the new conditions for 5-Rule, as follows:

The bracket variables are rejected if,

- $(\mu=v)$ or $(v=\gamma)$ or $(\mu=\gamma)$ and

- $(\mu, v) \in$ Rule $5 \neq(\mu, v) \in$ Rule4 .

Applying those conditions in formula (4), the row elements are uniquely defined, and the algorithm terminates with the correct matrix dimension.

Based on the concepts of divisors and algebraic geometry, a systematic approach for homogenising the polynomial equations have been realised and designed. Therefore, by Definition 2, the homogeneous coordinate for each support of system (7) is constructed for the case $h=k=1$,

\begin{tabular}{|c|c|}
\hline row & $\alpha \rightarrow \phi(\alpha)_{i}$ \\
\hline 1 & $(1,0) \rightarrow(1,0,0,2,2)$ \\
\hline 2 & $(1,0) \rightarrow(1,0,0,2,2)$ \\
\hline 3 & $(0,1) \rightarrow(0,1,2,2,0)$ \\
\hline 4 & $(0,1) \rightarrow(1,1,1,1,1)$ \\
\hline 5 & $(2,1) \rightarrow(2,1,0,0,2)$ \\
\hline 6 & $(1,2) \rightarrow(1,2,2,0,0)$ \\
\hline & column \\
\hline
\end{tabular}

These homogenisation coordinates are in $S_{\text {int }}(2 Q)$ and index the rows of the hybrid resultant matrix. In (4), given that $k \in R_{3}, j \in R_{2}$ and $i \in R_{1}$. The bracket variables are constructed. Starting with the first exponent, $(0$, 0) we have,

$$
\begin{array}{rcccccc}
(0,0) \rightarrow & 0 & 0 & 1 & 3 & 1 \\
& \vdots & \vdots & \vdots & \vdots & \vdots \\
& \alpha_{1} & \alpha_{2} & \alpha_{3} & \alpha_{4} & \alpha_{5}
\end{array}
$$


Continuing 5-Rule process, the empirical results for the combinations $(\mu, v, \gamma)$ of all points in $Q$, are shown in Table 2. Definition 3 is used on the construction of the exponent vectors of the facet variables. The algorithm terminates with the correct matrix dimension and the computational cost is $\mathcal{O}\left(n^{3}\right)$.

Table 2: The Combination $\mu, v, \gamma$ for all $\alpha \in Q$ when $h=k=1$

\begin{tabular}{|c|l|l|}
\hline$\alpha$ & $\left(\alpha_{1}, \ldots, \alpha_{5}\right)$ & \multicolumn{1}{|c|}{$(\mu, v, \gamma)$} \\
\hline$(0,0)$ & $(0,0,1,3,1)$ & 0 \\
$(1,0)$ & $(1,0,0,2,2)$ & $(2,4,1),(2,6,1),(5,3,1),(5,4,1),(5,6,1)$ \\
$(0,1)$ & $(0,1,2,2,0)$ & 0 \\
$(1,1)$ & $(1,1,1,1,1)$ & $(2,6,1),(4,6,1),(5,6,1),(2,4,3),(5,4,3),(2,6,3)$, \\
& & $(5,6,3)$ \\
$(2,1)$ & $(2,1,0,0,2)$ & $(2,5,3),(5,6,1)(5,3,4)(2,4,6),(5,6,2),(5,6,4)$ \\
$(1,2)$ & $(1,2,2,0,0)$ & $(2,6,3),(4,6,3),(5,6,3)$ \\
\hline
\end{tabular}

\section{MAIN RESULTS}

The results on the implementations of the hybrid Sylvester-Bèzout resultant matrix are generalised. The main theorem that established the conditions that can give a determinantal hybrid resultant formula for the class of unmixed bivariate polynomial systems is derived and proven.

Let $r, t$ be non-negative integers and $h, k$ be positive integers. Consider the unmixed polynomial system of three equations in two variables of the form,

$f_{i}=C_{i 1} x^{r} y^{t}+C_{i 2} x^{r+h} y^{t}+C_{i 3} x^{r} y^{t+k}+C_{i 4} x^{r+h} y^{t+k}+C_{i 5} x^{r+2 h} y^{t+k}$ $+C^{i 6} x^{r+h} y^{t+2 k}$,

with $i=1,2,3$ and the support of the system is

$$
A=\{(r, t),(r+h, t),(r, t+k),(r+h, t+k),(r+2 h, t+k),(r+h, t+2 k)\} .
$$


If $h=k$, the system (8) whose support is reduced to

$$
A=\{(r, t),(r+h, t),(r, t+k),(r,+2 h, t+k),(r+h, t+2 k)\} .
$$

Observing the results of the implementation gives the following theorem.

Theorem 4. Let $r, t \geq 0$, where $r, t \in \mathbf{Z}$ and let $h \in \mathbf{Z}^{+} \cdot$ Let $f_{1,} f_{2,}$ and $f_{3}$ be an unmixed polynomial system with support $A=\{(r, t),(r+h, t),(r, t+h),(r+$ $h, t+h),(r+2 h, t+h),(r+h, t+2 h)\}$. For each $i=1, \ldots, 6$ the homogeneous coordinate of $e_{i}$ for $r=t=0$ equals the homogeneous coordinate of $e_{i}$ for any $r>0$ or $t>0$.

Proof. The Newton polytope of $A$ and the respective normal vector $v_{\mathrm{i}}$ is shown in Fig. 2 where $v_{1}=(1,0), v_{2}=(0,1), v_{3}=(-1,1), v_{4}=(-1,-1)$, and $v_{5}=$ $(1,-1)$. From $(6)$ the convex hull of A is the Newton polytope $Q$ is defined by $a_{\mathrm{i}}=-\min _{m \in Q}\left\langle m, v_{i}\right\rangle$ and the Q-homogenisation map $\phi_{Q}: \mathrm{Z}^{2} \rightarrow \mathrm{Z}^{5}$ is defined by $\phi_{Q}(\alpha)_{i}=\left\langle\alpha, v_{i}\right\rangle+a_{i}$ for $i=1, \ldots, 5$.

Taking $(r, t)=0$, the homogeneous coordinates for each element of $A$,

$$
\begin{aligned}
& \phi_{Q}(0,0)=(0,0, h, 3 h, h)=h(0,0,1,3,1) \\
& \phi_{Q}(h, 0)=(h, 0,0,2 h, 2 h)=h(1,0,0,2,2) \\
& \phi_{Q}(0, h)=(0, h, 2 h, 2 h, 0)=h(0,1,2,2,0) \\
& \phi_{Q}(h, h)=(h, h, h, h, h)=h(1,1,1,1,1) \\
& \phi_{Q}(2 h, h)=(2 h, h, 0,0,2 h)=h(2,1,0,0,2) \\
& \phi_{Q}(h, 2 h)=(h, 2 h, 2 h, 0,0)=h(i, 2,2,0,0)
\end{aligned}
$$

Let $r, t>0$. Let $a=(r, t)$, then the homogeneous coordinates for each element of $A$,

$$
\begin{aligned}
\phi_{Q}(a)_{1} & =<(r, t),(1,0)>+(-r)=0 \\
\phi_{Q}(a)_{2} & =<(r, t),(0,1)>+(-r)=0 \\
\phi_{Q}(a)_{3} & =<(r, t),(-1,1)>+(r-t+h)=h \\
\phi_{Q}(a)_{4} & =<(r, t),(-1,-1)>+(r+t+3 h)=3 h \\
\phi_{Q}(a)_{5} & =<(r, t),(1,-1)>+(-r+t+h)+h \\
\therefore \phi_{Q}(r, t) & =(0,0, h, 3 h, h) .
\end{aligned}
$$


Continuing the same computation for other exponent vectors the following homogeneous coordinates are derived for each $a, \phi_{Q}(r+h, t)$ $=(h, 0,0,2 h, 2 h), \phi_{Q}(r, t+h)=(0, h, 2 h, 2 h, 0), \phi_{Q}(r+h, t+h)=(h, h, h, h, h)$, $\phi_{Q} \quad(r+2 h, t+h)=(2 h, h 0,0,2 h)$ and $\phi_{Q}(r+h, t+2 h)=(h, 2 h, 2 h, 0,0)$

Thus,

$$
\begin{aligned}
& \phi_{Q}(0,0)=\phi_{Q}(r, t)=(0,0,1,3,1) \\
& \phi_{Q}(h, 0)=\phi_{Q}(r+h, t)=(1,0,0,2,2) \\
& \phi_{Q}(0, h)=\phi_{Q}(r, t+h)=(0,1,2,2,0) \\
& \phi_{Q}(h, h)=\phi_{Q}(r+h, t+h)=(1,1,1,1,1) \\
& \phi_{Q}(2 h, h)=\phi_{Q}(r+2 h, t+h)=(2,1,0,0,2) \\
& \phi_{Q}(h, 2 h)=\phi_{Q}(r+h, t+2 h)=(1,2,2,0,0) .
\end{aligned}
$$

Theorem 5. Let $f_{1}, f_{2}$ and $f_{3}$ be an unmixed polynomial system with support $A=\{(0,0),(h, 0),(0, h),(h, h),(2 h, h),(h, 2 h)\}$. For each $i=1, \ldots, 6$ the homogeneous coordinate of $e_{1}$ for $h=1$, equals the homogeneous coordinate of $e_{1}$ for any $h>1$.

Proof. For $h=1$, name the support of the system $A_{1}$ given by $A=\{(0$ $, 0),(1,0),(0,1),(1,1),(2,1),(1,2)\}$. Homogeneous coordinate for each element of $A_{1} \phi_{Q}(0,0)=(0,0,1,3,1), \phi_{Q}(1,0)=(1,0,0,2,2), \phi_{Q}(0,1)=(0,1,2,2,0)$, $\phi_{Q}(1,1)=(1,1,1,1,1), \phi_{Q}(2,1)=(2,1,0,0,2), \phi_{Q}(1,2)=(1,2,2,0,0)$.

Taking $h>1$, the homogeneous coordinates of each element of $A_{\mathrm{h}}$ are $\phi_{Q}(h, 0)=h(1,0,0,2,2), \phi_{Q}(0, h)=h(0,1,2,2,0), \phi_{Q}(h, h)=h(1,1,1,1,1)$, $\phi_{Q}(2 h, h)=h(2,1,0,0,2), \phi_{Q}(h, 2 h)=h(1,2,2,0,0)$.

Theorem 4 illustrates that scaling the edges of $A_{1} h$ times gives the same homogeneous coordinate since for all $\hat{e}_{i} \in A_{h}, \phi\left(\hat{e}_{i}\right)=h \phi\left(e_{i}\right)=\phi\left(e_{i}\right)$ by Definition 1. Each homogeneous coordinate is a point in $\mathbf{P}^{4}$ which is written uniquely up to multiplication by $\lambda \in \mathbf{C}^{*}$. Here $\lambda=h$.

Example 2. Consider the following nonlinear unmixed system,

$$
f_{\mathrm{i}}=b_{i 1}+b_{i 2} x^{2}+b_{i 3} y^{2}+b_{i 4} x^{2} y^{2}+b_{15} x^{2} y^{2}, i=1,2,3 \text {. }
$$


This system is generated when $h=2(h>1)$ and gives support $A=$ $\{(0,0),(2,0),(0,2),(2,2),(4,2),(2,4)\}$. By Definition 2 , the homogeneous coordinates for each support are shown in Table 3.

Table 3: Homogeneous Coordinates

\begin{tabular}{|c|c|}
\hline $\begin{array}{c}\text { Exponent vector } \\
a\end{array}$ & $\begin{array}{c}\text { Homogeneous coordinate } \\
\left(a_{1}, \ldots, a_{5}\right)\end{array}$ \\
\hline$(0,0)$ & $(0,0,2,6,2)=2(0,0,1,3,1)$ \\
\hline$(2,0)$ & $(2,0,0,4,4)=2(1,0,0,2,2)$ \\
\hline$(0,2)$ & $(0,2,4,4,0)=2(0,1,2,2,0)$ \\
\hline$(2,2)$ & $(2,2,2,2,2)=2(1,1,1,1,1)$ \\
\hline$(4,2)$ & $(4,2,0,0,4)=2(2,1,0,0,2)$ \\
\hline$(2,4)$ & $(2,4,4,0,0)=2(1,2,2,0,0)$ \\
\hline
\end{tabular}

This example showed that by scaling the edges, $h=2$ times gives the same homogeneous coordinate for $h=1$.

\section{CONCLUSION}

The concepts in algebraic geometry were highlighted through the construction of Bèzout matrix. The implementation of the algorithm of Bèzout matrix is on the unmixed bivariate polynomial systems of the form equation (8) by considering the origin as the distinguished point of the Newton polytope. Detail implementation and construction are presented to indicate that, it is sufficient to consider only the case when $r=t=0$ and $h=k$ and scaling the edges of $A_{1}$, h times gives the same homogeneous coordinate.

\section{ACKNOWLEDGEMENT}

A part of this research is supported by research grant Bestari(GBEST-1/2019), Universiti Teknologi MARA, Johor Branch and received collaboration from UTM Skudai, Malaysia by Research University Grant vote number Q.J130000.7126.04J05, Ministry of Higher Education (MOHE) Malaysia. 


\section{REFERENCES}

[1] M.J. Puente, 2002. Real plane algebraic curves, Expositiones Mathematicae, 20(4), pp. 291-314. https://doi.org/10.1016/S07230869(02)80009-3

[2] I. Karzhemanov, "On the cut-and-paste property of algebraic varieties," Mathematics, Algebraic Geometry, arXiv.org (2014):1411.6084v3, http://arxiv.org/pdf/1411.6084.pdf.

[3] I. Karzhemanov, "On characterization of toric varieties," Mathematics, Algebraic Geometry, arXiv.org (2016):1306.4131v9, http://arxiv.org/ pdf/1306.4131.pdf.

[4] D.A. Cox, "What is a Toric Variety? Topics in algebraic geometry and geometric modeling," in Contemporary Mathematics, Amer. Math. Soc., Providence, RI., 334, 2003, pp. 203 - 223.

[5] D.A. Cox, J. Little, and H.K. Schenck, Toric Varieties, Graduate Studies in Mathematics, Amer. Math. Soc., Providence, RI., 124, 2011.

[6] T.W. Sederberg, 1997. Application to Computer Aided Geometric Design, in D. Cox and B. Sturmfels (eds) Applications of Computational Algebraic Geometry: Short Course, San Diego, California, AMS, Rhode Island, USA, pp. 67-89.

[7] D. A. Cox, J. Little, and D. O'Shea, Using Algebraic Geometry, Graduate Texts in Mathematics, Springer-Verlag, New York, vol. 185, 2005.

[8] A. Dickenstein, I. Z. Emiris, and V. Fisikopoulos, "Combinatorics of 4-Dimensional Resultant Polytopes," ISSAC'13 Proc. of the $38^{\text {th }}$ Int. Symp. on Symb. Algebraic Computation, ACM, New York, USA, 2013, pp. $173-180$.

[9] S. N. Ahmad and A. Aris, "The Implementation of a Hybrid Resultant Matrix formulation," in American Institute of Physics Journal, AIP Conference Proceedings, 1682, 2015, pp. 020015 
SCIENTIFIC RESEARCH JOURNAL

[10] A. Khetan, "The resultant of an unmixed bivariate system," Journal of Symbolic Computation, 36, pp. 425 - 442, 2002. 